\title{
Volumetric MRI analysis of brain structures in patients with history of first and repeated suicide attempts: a cross sectional study
}

\section{Milda Sarkinaite ( $\sim$ milda.sarkinaite@gmail.com )}

Lietuvos sveikatos mokslu universitetas https://orcid.org/0000-0002-9394-2624

\section{Rymante Gleizniene}

Lietuvos sveikatos mokslu universitetas

\section{Virginija Adomaitiene}

Lietuvos sveikatos mokslu universitetas

\section{Kristina Dambrauskiene}

Lietuvos sveikatos mokslu universitetas

\section{Nijole Raskauskiene}

Lietuvos sveikatos mokslu universitetas

\section{Vesta Steibliene}

Lietuvos sveikatos mokslu universitetas

\section{Research article}

Keywords: suicide attempt, Free Surfer, magnetic resonance imaging, hippocampus, frontal cortex, temporal cortex

Posted Date: August 10th, 2020

DOl: https://doi.org/10.21203/rs.3.rs-36981/v2

License: (c) (1) This work is licensed under a Creative Commons Attribution 4.0 International License. Read Full License

Version of Record: A version of this preprint was published at Diagnostics on March 10th, 2021. See the published version at https://doi.org/10.3390/diagnostics11030488. 


\section{Abstract}

Background Structural brain changes are found in suicide attempters, as well as in patients with mental disorders. It remains unclear whether the suicidal behavior is related to atrophy of brain regions and how the morphology of specific brain areas is changing with each suicide attempt. This cross-sectional study examined volumetric differences in brain regions among patients with history of first and repeated suicide attempts in comparison to healthy controls (HC). Methods The sample consisted of 56 adults, non-psychotic patients without cognitive impairment and any organic brain disorders hospitalized after first suicide attempt (first $S A)(n=29)$ and more than one suicide attempt (SA>1) during the lifetime $(n=27)$; and 54 adult volunteers without history of mental disorder and suicide attempts, designated as HC. The MRI data were collected using 1.5 T Siemens Avanto scanner. Brain cortical thickness, grey and white matter volumes were measured using FreeSurfer 6.0 automatic segmentation technique. Results In comparison to $\mathrm{HC}$, patients with first SA had 3.5, 3.58 and $4.19 \%$ significantly lower mean cortical thickness of the superior and rostral middle frontal areas of the left hemisphere and superior frontal area of the right hemisphere, respectively; 4.09, 4.02 and 4.49\% lower mean cortical thickness of the inferior, middle and superior temporal areas of the left hemisphere, respectively. In comparison to HC, patients after $S A>1$ had a significantly lower mean cortical thickness (from 4.02 to $8.33 \%$ ) in ten areas of frontal cortex of the left hemisphere and seven areas of the right hemisphere; from 3.90 to $6.04 \%$ difference in six areas of temporal cortex in both hemispheres. The comparison of hippocampus volume showed a significantly lower mean volume (7.86 to $9.89 \%$ ) of left and right parts in patients with $S A>1$, but not in patients with first SA. Conclusions Hospitalized suicide attempters had lower frontal and temporal cortical thickness and smaller parts of hippocampus than HC; these differences were significantly higher in repeated suicide attempters than in patients with first SA. Our findings suggest that repeated suicidal behavior is associated with intensifying atrophy of specific brain structures, independently of diagnosis of depressive disorders.

\section{Background}

According to the World Health Organization, globally, 793000 suicide occurrences are recorded each year (10.5 per 100000 population), and there are from two to three times more suicide attempts [1]. In Lithuania, suicide is one of the major public health issues. It should be noted that with the rate of $31,9 / 10000$ Lithuania is in the first place in the European Union (EU) in terms of the number of suicides. It is three times higher compared to the rest of the world and twice as high as recorded in the EU $(15,4$ per 100000 population) [1]. Therefore, in Lithuania, as well as in the whole world, great importance is attached to identifying both psychosocial and neurobiological factors of suicidal behavior for the purpose of effective suicide prevention.

A number of neurobiological models have been proposed to explain the suicidal behavior [2]. The identification of these models could be the effective step in suicide prevention. Nowadays structural neuroimaging is one of the best modern methods for examinations of suicidal behavior in the living brain [4]. There are more than 30 studies known which indicate that structural brain changes are found in 
suicide attempters, they also reveal how variation in brain volume is associated with pathogenesis of suicide [3]. However, data are quite contradictory; brain changes in most studies are more related to mental disorders than suicidal behavior [3]. Atrophy of dorsolateral (DL) prefrontal cortex (PFC), ventrolateral (VL) areas of PFC and anterior cingulate cortex (ACC) is found in suicide attempters, the patients with depression or schizophrenia $[4,5]$. Other studies on suicidality detected grey matter atrophy in the left superior temporal gyrus, left orbitofrontal cortex (OFC), superior left parietal lobe, thalamus, insula of the right side, and some frontal regions [5-8]. In contrast, increased volumes of the right amygdala and the reciprocal inferior frontal white matter are also discovered in suicide attempters [5-8]. Research on suicide behavior in patients with unipolar disorder found support to volume reduction in right and left OFC, hippocampal volume in comparison to healthy controls [9] .

To the best of our knowledge, there has been no investigation into brain volume changes after first and repeated SA. Thus, it is remains unclear, how the morphology of specific brain areas changes with each subsequent suicide attempt. In this cross-sectional study we examined structural differences in temporal cortex (TC), frontal cortex and hippocampal regions between patients with history of first and repeated $\mathrm{SA}$ in comparison to healthy controls by using brain magnetic resonance imaging (MRI) and automatic segmentation technique.

\section{Methods}

\subsection{Study population and settings}

Adults, non-psychotic patients, men and women, without cognitive impairment, any other organic brain disorders, or severe unstable medical conditions, hospitalized after SA into the Psychiatry department during a six-month period, eligible for MRI scan were invited to participate in this cross-sectional study. Psychiatric diagnoses and suicide attempts were evaluated by a psychiatrist and defined under the Diagnostic and Statistical Manual of Mental Disorders 5th edition (DSM-5) [10] . SA has been defined as a nonfatal self-directed, potentially injurious behavior with any intent to die as a result of the behavior [11]. The control group consisted of adult subjects (men and women), who consecutively came for preventive check-ups to a family physician at the Clinic of Family Medicine. The exclusion criteria for control group were the anamnesis of mental disorder, alcohol or drug abuse or dependence, anamnesis of $\mathrm{SA}$, cognitive impairment, severe unstable medical conditions or any organic brain disorders.

The study protocol and informed consent procedures were approved by the Bioethics Committee at the Lithuanian University of Health Sciences, Kaunas, Lithuania (2017-06-23, No. BEC-LSMUCR-67). The investigation was carried out in accordance with the Declaration of Helsinki. Each study participant gave written informed consent prior to all study procedures.

Of all invited as study patients, 59 subjects (response rate 92\%) agreed to participate in the study. One patient was excluded due to specific MRI changes of carbon monoxide poisoning and 2 patients were excluded due to anamnesis of chronic alcohol consumption that can cause brain changes. The HC group included 54 volunteers (response rate $68 \%$ ). Hence, the final study sample consisted of 56 study patients 
(mean age 40.8 (16.8) years, 35.2\% of them were men) after SA and 54 HC (mean age 41.0 (16.3) years, $39.3 \%$ were men). There were no significant differences between patients and controls with regard to age and gender (Table 1). All study patients filled the sociodemographic questionnaire and data about the history of suicide attempts (for full details, see Additional file 1).

\subsection{MRI processing and analysis}

Patients after SA and HC went through the same MRI protocol, which consisted of T2weighted(T2W)/FLAIR axial (TR 9000ms, TE 89ms) and T2W/fl2d/hemo axial (TR 800ms, TE26ms) sequences in order to rule out the pathologies as lesions and haemorrhage. Image data were collected using 1.5 Tesla ( $\mathrm{T}$ ) Siemens Avanto scanner with a standard quadrature head volume coil. Head movement was prevented by pads inside the coil. Whole-brain T1-weighted (T1W) mpr/p2/iso axial (TR 1900ms, TE 3.35ms, 1 mm slice thickness) sequence was used for volumetric measurement. During brain MRI, the patients had no suicidal ideation.

All MRI data were subject to strict quality control procedures. First, the radiologists checked T1W images for artefacts. If the latter met the requirements, they were converted to .mgz files. Brain cortical thickness and grey- and white-matter volumes were measured using FreeSurfer 6.0 image analysis suite ((http://surfer.nmr.mgh.harvard.edu/). This automatic reconstruction technique outweighs standard brain volume measurement due to the fact that it investigates across the whole brain and can locate changes which may be very small or do not match usual anatomical subdivisions. The FreeSurfer software constructs a three-dimensional representation of the cortical surface and volume quantifications applying image intensities and continuity data from the entire MRI volume to create reproductions of the border between gray and white matter and pial surface [12]. FreeSurfer's Desikan-Killiany atlas was used to define regions of interest for the brain cortex: the frontal cortex contained 11 regions, temporal cortex - 9 regions [13]. Freesurfer's automated segmentation of hippocampal substructures divided the hippocampus into 13 subfields [14].

\subsection{Statistical analysis}

The Shapiro-Wilk test was employed to verify whether the data meet the conditions of the normal distribution. The comparison between all patients after SA and comparison control subjects was performed using two-sample t-tests. Observed differences were considered statistically significant when the calculated significance level ( $p$-value) was lower than the selected significance level $(a=0.05)$. Values for the average cortical thickness were analyzed using a general linear model controlling for the effect of age. ANOVA was used to test the equality of three group means, statistically significant results indicate that not all of the group means are equal. Post hoc tests were used to explore differences between multiple group means. Frontal and TC thickness as well as hippocampus volume was compared among the three groups ( $\mathrm{HC}$, first $\mathrm{SA}, \mathrm{SA}>1$ ) using analysis of covariance (ANCOVA), with post hoc pairwise comparisons for age as covariate of interest. The $p$-value was used in the ANOVA output to determine whether the differences between some of the means are statistically significant. F values are based on the pairwise comparisons among the means. P-values were adjusted for multiple comparisons using 
Bonferroni correction for multiple comparisons. An additional entire cortex analysis was performed in order to investigate a potential gender by groups interaction in cortical thickness. Covarying for gender in the full-group analyses did affect the results. Thus, the final analyses were performed without covarying for gender. Alpha levels for statistical significance testing were set to 0.05. IBM SPSS Statistics 23 and Microsoft Excel 2010 were used for statistical analysis.

\section{Results}

All study patients, hospitalized after SA ( $n=56)$ were divided into the following two groups: suicide attempters with a history of at least one suicide attempt (first SA group, $\mathrm{n}=29$ ), and suicide attempters who reported more than one suicide attempt during the lifetime ( $S A>1$ group, $n=27$ ). As reported in Table 1 , the groups did not differ significantly in age, gender, psychiatric diagnoses or method of suicide attempt, but they did vary in terms of marital status, with a greater proportion of single participants in the first SA group.

Table 1 Demographic characteristics of all study subjects.

\begin{tabular}{|c|c|c|c|c|c|c|}
\hline & $\begin{array}{c}\mathrm{HC} \\
(\mathrm{n}=54)\end{array}$ & after SA $(n=56)$ & $\mathrm{p}^{\mathrm{a}}$ & $\begin{array}{l}\text { First SA grou } \\
\qquad(\mathrm{n}=29)\end{array}$ & $\begin{array}{l}\text { SA }>1 \text { group } \\
\quad(n=27)\end{array}$ & $\begin{array}{l}\mathrm{F} / \mathrm{p} \\
\mathrm{c}^{2}\end{array}$ \\
\hline $\begin{array}{l}\text { Age mean }(\mathrm{SD}) \text {, } \\
\text { years range }\end{array}$ & 41.0 (16.3) 18-77 & 40.8 (16.8) 18-74 & 0.955 & $41.1(18.1)$ & $40.6(15.6)$ & $0.993^{\mathrm{a}}$ \\
\hline Gender, & $19 / 35(35.2 / 64.8)$ & $22 / 34$ & 0.656 & $14 / 15$ & $8 / 19$ & $0.320^{\mathrm{a}}$ \\
\hline $\mathrm{M} / \mathrm{F}, \mathrm{n}(\%)$ & & $(39.3 / 60.7)$ & & $48.3 / 51.7$ & 29.6/70.4 & \\
\hline Residential area, rural/urban, n (\%) & & $11 / 45(19.6 / 80.4)$ & & $\begin{array}{c}3 / 26 \\
10.3 / 89.7\end{array}$ & $\begin{array}{c}8 / 19 \\
29.6 / 70.4\end{array}$ & 0.070 \\
\hline Marital status, married/single, n (\%) & & $\begin{array}{c}27 / 29 \\
(48.2 / 51.8)\end{array}$ & & $\begin{array}{c}10 / 19 \\
34.5 / 65.5\end{array}$ & $\begin{array}{l}17 / 10 \\
63 / 37\end{array}$ & 0.033 \\
\hline $\begin{array}{l}\text { Psychiatric diagnosis, } \\
\text { MDD/ otherb }{ }^{b}, \mathrm{n}(\%)\end{array}$ & & $21 / 35(37.5 / 62.5)$ & & $\begin{array}{c}10 / 19 \\
34.5 / 65.5\end{array}$ & $\begin{array}{c}11 / 16 \\
40.7 / 59.3\end{array}$ & 0.418 \\
\hline Age at first SA, years & & $38.2(17.2)$ & & $41.1(18.1)$ & $35.1(15.9)$ & 0.201 \\
\hline $\begin{array}{l}\text { History of SA, } \\
\text { First time/repeated, n (\%) }\end{array}$ & & $\begin{array}{c}29 / 27 \\
(51.8 / 48.2)\end{array}$ & & - & - & \\
\hline Method of SA, drugs/physical, n (\%) & & $31 / 25(55.4 / 44.6)$ & & $\begin{array}{c}16 / 13 \\
55.2 / 44.8\end{array}$ & $\begin{array}{c}15 / 12 \\
55.6 / 44.4\end{array}$ & 0.595 \\
\hline $\begin{array}{l}\text { Alcohol intoxication during SA, } \\
\text { Yes/no, n (\%) }\end{array}$ & & $\begin{array}{c}22 / 34 \\
(39.3 / 60.7)\end{array}$ & & $\begin{array}{c}12 / 17 \\
41.4 / 58.6\end{array}$ & $\begin{array}{l}10 / 17 \\
37 / 63\end{array}$ & 0.477 \\
\hline
\end{tabular}

HC, Healthy controls; SA, Suicide attempt; SD, Standard deviation; M, Male; F, Female; MDD, major depressive disorder;

$\mathrm{p}^{\mathrm{a}}$ value between three groups: HC, first SA, SA>1.

${ }^{\mathrm{b}}$ other diagnoses include: anxiety disorder, personality disorder, adjustment disorder.

The volumetric analysis of all brain structures was done. Overall, significant differences between all SA patients and healthy controls groups were found in frontal and TC thickness and in volume of the left and 
right parts of hippocampus. Decreased cortical thicknesses were observed in both hemispheres. Analysis of other brain regions did not reveal significant results.

The analysis of frontal cortex thickness is shown in Table 2. The comparison between the patients after first SA and HC revealed the significant lower cortical thickness of the superior frontal $(p=0.041)$ and rostral middle frontal $(p=0.016)$ areas of the left hemisphere and superior frontal area $(p=0.021)$ of the right hemisphere, with relative 3.5, 3.58 and $4.19 \%$ difference of mean cortical thickness in these cortex areas, respectively. Moreover, the comparison between the $S A>1$ group and controls revealed the significant lower frontal cortical thickness in ten areas of the left hemisphere and seven areas of the right hemisphere, with relatively higher difference (4.02 to $8.33 \%$ ) of the mean cortical thickness between these two groups. Differences in four of frontal cortex regions were highly significant $(p<0.0001)$ in patients with $S A>1$ as compared with the control group.

Table 2 The significant differences in means of frontal cortex thickness between the patients after first suicide attempt, repeated suicide attempts and study controls (HC, first SA and SA $>1$ ).

\begin{tabular}{|c|c|c|c|c|c|c|c|c|c|c|}
\hline \multirow[t]{2}{*}{ Hemisphere } & \multirow[t]{2}{*}{ Frontal cortex part } & \multicolumn{7}{|c|}{ Mean cortical thickness (95\% CI) in mm } & \multicolumn{2}{|c|}{$\begin{array}{c}\text { ANCOVA } \\
\text { age adjusted }\end{array}$} \\
\hline & & $\begin{array}{c}\mathrm{HC} \\
(\mathrm{n}=54)\end{array}$ & $\begin{array}{c}\text { First SA } \\
(\mathrm{n}=29)\end{array}$ & Post-hoc & $\%$ diff & $\begin{array}{l}\mathrm{SA}>1 \\
(\mathrm{n}=27)\end{array}$ & Post-hoc & $\%$ diff. & & \\
\hline & & 1 & 2 & 1 vs. 2 & $1-2$ & 3 & 1 vs 3 & $1-3$ & $\mathrm{~F}_{2.106}$ & $\mathrm{p}$ \\
\hline \multicolumn{11}{|l|}{ Left } \\
\hline & Lateral orbitofrontal & $2.79(2.75-2.83)$ & $2.72(2.66-2.77)$ & 0.140 & 2.50 & $2.66(2.60-2.72)$ & 0.002 & 4.65 & 6.71 & 0.002 \\
\hline & Medial orbitofrontal & $2.55(2.50-2.58)$ & $2.54(2.49-2.60)$ & 1.000 & 0.39 & $2.42(2.36-2.48)$ & 0.003 & 5.09 & 6.74 & 0.002 \\
\hline & Pars opercularis & $2.66(2.61-2.72)$ & $2.58(2.51-2.65)$ & 0.149 & 3.00 & $2.48(2.41-2.55)$ & $<0.001$ & 6.77 & 11.29 & $<0.001$ \\
\hline & Pars orbitalis & $2.88(2.82-2.94)$ & $2.78(2.70-2.86)$ & 0.142 & 3.47 & $2.69(2.61-2.77)$ & 0.001 & 6.59 & 7.32 & 0.001 \\
\hline & Pars triangularis & $2.56(2.51-2.61)$ & $2.49(2.43-2.56)$ & 0.329 & 2.73 & $2.41(2.34-2.48)$ & 0.002 & 5.85 & 6.40 & 0.002 \\
\hline & Precentral & $2.52(2.46-2.56)$ & $2.42(2.35-2.48)$ & 0.093 & 3.96 & $2.31(2.24-2.38)$ & $<0.001$ & 8.33 & 10.34 & $<0.001$ \\
\hline & Rostral middle frontal & $2.51(2.47-2.54)$ & $2.42(2.37-2.47)$ & 0.016 & 3.58 & $2.37(2.32-2.42)$ & $<0.001$ & 5.58 & 11.48 & $<0.001$ \\
\hline & Superior frontal & $2.85(2.81-2.90)$ & $2.75(2.68-2.81)$ & 0.041 & 3.50 & $2.66(2.59-2.73)$ & $<0.001$ & 6.67 & 11.11 & $<0.001$ \\
\hline & Frontal pole & $2.84(2.76-2.91)$ & $2.76(2.65-2.87)$ & 0.765 & 2.81 & $2.66(2.55-2.77)$ & 0.032 & 6.33 & 3.43 & 0.036 \\
\hline & Caudal middle frontal & $2.64(2.59-2.69)$ & $2.54(2.48-2.61)$ & 0.058 & 3.78 & $2.50(2.43-2.69)$ & 0.002 & 5.3 & 6.78 & 0.002 \\
\hline \multicolumn{11}{|l|}{ Right } \\
\hline & Lateral orbitofrontal & $2.73(2.68-2.77)$ & $2.69(2.63-2.75)$ & 0.944 & 1.46 & $2.62(2.56-2.68)$ & 0.013 & 4.02 & 4.28 & 0.016 \\
\hline & Pars opercularis & $2.63(2.57-2.68)$ & $2.57(2.49-2.65)$ & 0.824 & 2.28 & $2.48(2.40-2.56)$ & 0.011 & 5.70 & 4.39 & 0.015 \\
\hline & Pars orbitalis & $2.86(2.79-2.91)$ & $2.81(2.72-2.89)$ & 1.000 & 1.74 & $2.71(2.62-2.80)$ & 0.026 & 5.24 & 3.58 & 0.031 \\
\hline & Pars triangularis & $2.55(2.49-2.60)$ & $2.51(2.44-2.58)$ & 1.000 & 1.56 & $2.42(2.35-2.49)$ & 0.016 & 5.09 & 4.07 & 0.020 \\
\hline & Precentral & $2.46(2.41-2.51)$ & $2.39(2.32-2.46)$ & 0.290 & 2.84 & $2.31(2.24-2.38)$ & 0.002 & 6.09 & 6.42 & 0.002 \\
\hline & Rostral middle frontal & $2.46(2.41-2.50)$ & $2.42(2.36-2.49)$ & 1.000 & 1.62 & $2.35(2.92-2.42)$ & 0.040 & 4.47 & 3.19 & 0.045 \\
\hline & Superior Frontal & $2.86(2.81-2.91)$ & $2.74(2.68-2.81)$ & 0.021 & 4.19 & $2.68(2.62-2.75)$ & $<0.001$ & 6.29 & 9.58 & $<0.001$ \\
\hline & Caudal middle frontal & $2.59(2.54-2.64)$ & $2.51(2.44-2.58)$ & 0.267 & 3.08 & $2.48(2.40-2.55)$ & 0.054 & 4.24 & 3,33 & 0.039 \\
\hline
\end{tabular}

HC, healthy controls; SA, Suicide attempt.

F, p-values are based on the pairwise comparisons among the means (three groups: HC, first SA, SA>1).

$\%$ diff - relative difference describes the difference of the mean cortical thickness between two groups: HC and patients after SA.

Significant p-values $(\mathrm{p}<0.05)$ are indicated in bold. 
Table 3 presented the comparison of thickness of temporal and frontal cortex among study groups. Patients after first SA, in comparison to $\mathrm{HC}$, had significantly lower cortical thickness of the inferior $(p=0.002)$, middle $(p=0.013)$ and superior $(p=0.006)$ temporal areas of the left hemisphere, with relative $4.09,4.02$ and $4.49 \%$ difference of mean cortical thickness in these cortex areas, respectively. In patients with $S A>1$, in comparison to controls, the significant lower TC thickness was observed in the six areas of both hemispheres, with a difference (3.90 to $6.04 \%$ ) of the mean cortical thickness between these two groups. Differences in the inferior and middle temporal areas of the left hemisphere were observed as highly significant $(p<0.001)$.

Table 3 The significant differences in means of temporal cortex thickness between the patients after first suicide attempt, repeated suicide attempts and study controls (HC, first SA and SA>1)

\begin{tabular}{|c|c|c|c|c|c|c|c|c|c|c|}
\hline \multirow[t]{3}{*}{ Hemisphere } & \multirow[t]{3}{*}{ Temporal cortex part } & \multicolumn{7}{|c|}{ Mean cortical thickness $(95 \% \mathrm{CI})$ in $\mathrm{mm}$} & \multirow{2}{*}{\multicolumn{2}{|c|}{$\begin{array}{c}\text { ANCOVA } \\
\text { age adjusted, }\end{array}$}} \\
\hline & & \multirow{2}{*}{$\begin{array}{c}\begin{array}{c}\mathrm{HC} \\
(\mathrm{n}=54)\end{array} \\
1\end{array}$} & \multirow{2}{*}{$\begin{array}{c}\begin{array}{c}\text { First SA } \\
(\mathrm{n}=29)\end{array} \\
2\end{array}$} & \multicolumn{2}{|c|}{ Post-hoc \% diff. } & $\begin{array}{c}\mathrm{SA}> \\
(\mathrm{n}=27)\end{array}$ & Post-hoc & $\%$ diff. & & \\
\hline & & & & 1 vs. 2 & $1-2$ & 3 & 1 vs 3 & $1-3$ & $\mathrm{~F}_{2.106}$ & $\mathrm{p}$ \\
\hline \multirow[t]{5}{*}{ Left } & Fusiform & $2.78(2.73-2.83)$ & $2.73(2.66-2.80)$ & 0.573 & 1.79 & $2.67(2.60-2.74)$ & 0.031 & 3.90 & 3.53 & 0.033 \\
\hline & Inferior temporal & $2.93(2.89-2.98)$ & $2.81(2.75-2.87)$ & 0.002 & 4.09 & $2.78(2.72-2.83)$ & $<0.001$ & 5.01 & 11.55 & $<0.001$ \\
\hline & Middle temporal & $2.98(2.93-3.03)$ & $2.86(2.80-2.93)$ & 0.013 & 4.02 & $2.80(2.73-2.86)$ & $<0.001$ & 6.04 & 11.29 & $<0.001$ \\
\hline & Superior temporal & $2.89(2.84-2.93)$ & $2.76(2.70-2.83)$ & 0.006 & 4.49 & $2.76(2.69-2.82)$ & 0.005 & 4.49 & 7.61 & 0.001 \\
\hline & Temporal pole & 3.76 (3.67-3.85) & 3.59 (3.47-3.72) & 0.100 & 4.52 & $3.54(3.41-3.66)$ & 0.015 & 5.85 & 4.88 & 0.009 \\
\hline Right & Superior temporal & 2.89 (2.84-2.95) & $2.85(2.77-2.92)$ & 0.995 & 1.38 & $2.77(2.69-2.85)$ & 0.036 & 4.15 & 3.27 & 0.042 \\
\hline
\end{tabular}

HC, healthy controls; SA, Suicide attempt.

F, p-values are based on the pairwise comparisons among the means (three groups: HC, first SA, SA $>1$ ).

$\%$ diff - relative difference describes the difference of the mean cortical thickness between two groups: HC and patients after SA. Significant pvalues $(\mathrm{p}<0.05)$ are indicated in bold.

Significant differences in volume of left and right parts of hippocampus between study controls and all study patients are demonstrated in Table 4.

Table 4 The significant differences in mean volume of hippocampus parts between HC and all study patients 


\begin{tabular}{|c|c|c|c|c|c|c|}
\hline \multirow[t]{3}{*}{ Hemisphere } & \multirow[t]{3}{*}{ Part of hippocampus } & \multicolumn{3}{|c|}{ Mean volume $(95 \% \mathrm{CI})$ in $\mathrm{mm}^{3}$} & \multicolumn{2}{|c|}{$\begin{array}{c}\text { ANCOVA } \\
\text { age adjusted }\end{array}$} \\
\hline & & $\begin{array}{c}\mathrm{HC} \\
(\mathrm{n}=54)\end{array}$ & $\begin{array}{l}\text { All patients after SA } \\
\qquad(\mathrm{n}=56)\end{array}$ & $\%$ diff & & \\
\hline & & 1 & 2 & $1-2$ & $\mathrm{~F}_{1.107}$ & $\mathrm{p}$ \\
\hline \multicolumn{7}{|l|}{ Left } \\
\hline & ML & $564.42(545.31-583.55)$ & $536.10(517.28-554.93)$ & 5.01 & 2.97 & 0.039 \\
\hline & GC-DG & $292.54(282.38-302.68)$ & 273.57 (263.63-283.52) & 6.48 & 3.79 & 0.009 \\
\hline & CA3 & $195.78(188.04-203.51)$ & $183.84(176.24-191.43)$ & 6.09 & 4.77 & 0.031 \\
\hline & CA4 & $250.55(241.90-259.22)$ & $236.85(228.38-245.32)$ & 5.46 & 5.05 & 0.027 \\
\hline & HATA & $58.31(55.76-60.87)$ & $52.94(50.44-55.44)$ & 9.20 & 4.46 & 0.004 \\
\hline \multicolumn{7}{|l|}{ Right } \\
\hline & Subiculum & $428.21(411.15-445.27)$ & $404 . .3(387.15-420.91)$ & 5.58 & 3.35 & 0.049 \\
\hline & CA1 & 640.27 (616.07-664.45) & $605.72(581.90-629.54)$ & 5.39 & 4.06 & 0.047 \\
\hline & ML & 574.35 (553.83-594.88) & $545.01(524.75-565.26)$ & 5.10 & 3.09 & 0.047 \\
\hline & GC-DG & $303.14(292.17-314.10)$ & $287.19(276.44-297.94)$ & 5.26 & 4.25 & 0.042 \\
\hline & HATA & $61.01(58.18-63.84)$ & 56.54 (53.76-59.31) & 7.32 & 5.02 & 0.027 \\
\hline
\end{tabular}

HC, healthy controls; SA, Suicide attempt; ML, molecular layer; GC-DG, granule cell layer of dentate gyrus; CA, cornu amonis; HATA, hippocampus-amygdala transition area.

F, p - values are based on the pairwise comparisons among the means (two groups: HC and all patients after SA).

$\%$ diff - relative difference describes the difference of the mean hippocampus volume between two study groups: HC and patients after SA. Significant $\mathrm{p}$-values $(\mathrm{p}<0.05)$ are indicated in bold.

Analysis of patients with history of first SA did not reveal significant differences in volume of hippocampus parts, in comparison to $\mathrm{HC}$ (Table 5). However, the comparison of controls to patients with $S A>1$ reported significant differences in mean volume of left and right parts of hippocampus volume, with 7.86 to $9.89 \%$ relative differences of the mean hippocampus volume between two groups.

Table 5 The significant differences in hippocampus part mean volume between the patients after first suicide attempt, repeated suicide attempts and study controls (first SA, SA>1 and HC)

\begin{tabular}{|c|c|c|c|c|c|c|c|c|c|c|}
\hline \multirow[t]{2}{*}{ Hemisphere } & \multirow{2}{*}{$\begin{array}{c}\text { Part of } \\
\text { hippocampus }\end{array}$} & \multicolumn{7}{|c|}{ Mean volume $(95 \% \mathrm{CI})$ in $\mathrm{mm}^{3}$} & \multicolumn{2}{|c|}{$\begin{array}{c}\text { ANOVA } \\
\text { age adjusted }\end{array}$} \\
\hline & & $\mathrm{HC}(\mathrm{n}=54)$ & $\begin{array}{l}\text { First SA } \\
(\mathrm{n}=29)\end{array}$ & $\begin{array}{l}\text { Post- } \\
\text { hoc }\end{array}$ & $\begin{array}{c}\% \\
\text { diff. }\end{array}$ & $\mathrm{SA}>1(\mathrm{n}=27)$ & $\begin{array}{l}\text { Post- } \\
\text { hoc }\end{array}$ & $\begin{array}{c}\% \\
\text { diff. }\end{array}$ & & \\
\hline & & 1 & 2 & 1 vs. 2 & $1-2$ & 3 & 1 vs. 3 & $1-3$ & $\mathrm{~F}_{2.106}$ & $\mathrm{p}$ \\
\hline \multicolumn{11}{|l|}{ Left } \\
\hline & GC-DG & $\begin{array}{c}292.54(282.38- \\
302.68)\end{array}$ & $\begin{array}{c}277.34(263.49- \\
291.19)\end{array}$ & 0.247 & 5.19 & $\begin{array}{c}269.52(255.17- \\
283.88)\end{array}$ & 0.032 & 7.86 & 3.79 & 0.026 \\
\hline & HATA & $58.31(55.76-60.87)$ & $53.32(49.83-56.81)$ & 0.072 & 8.56 & $52.54(48.92-56.15)$ & 0.033 & 9.89 & 4.46 & 0.014 \\
\hline \multicolumn{11}{|l|}{ Right } \\
\hline & Subiculum & $\begin{array}{c}428.21(411.15- \\
445.27)\end{array}$ & $\begin{array}{c}417.39(394.12- \\
440.67)\end{array}$ & 1.000 & 2.52 & $\begin{array}{c}389.67(365.54- \\
413.80)\end{array}$ & 0.043 & 9.00 & 3.35 & 0.039 \\
\hline
\end{tabular}

HC, healthy controls; SA, Suicide attempt; GC-DG, granule cell layer of dentate gyrus; HATA, hippocampus-amygdala- transition-area. 
F, p - values are based on the pairwise comparisons among the means (three groups: HC, first SA, SA $>1$ ).

$\%$ diff - relative difference describes the difference of the mean hippocampus volume between two groups.

Significant $\mathrm{p}$-values $(\mathrm{p}<0.05)$ are indicated in bold.

Post-hoc analyses revealed no significant within group differences in brain thickness and volume of parts of the hippocampus in patients after SA, when comparing first SA group $(n=29)$ to $S A>1$ group $(n=27)$.

The comparison of frontal and temporal cortex thickness and the volumes of hippocampus parts of all study patients with history of SA according to psychiatric diagnoses (major depressive disorder vs. other diagnosis), method of suicidal attempt (intoxication with drugs $v s$. physical suicidal act) and acute alcohol intoxication before SA (yes vs. no) did not reveal significant differences.

\section{Discussion}

To our knowledge, it is the first study, which compared volumetric brain parameters in acute suicidal patients after first and repeated suicidal attempts to healthy subjects without a history of suicidal attempt and mental disorder. The main finding of this research that already after the first suicide attempt in comparison to healthy controls lower cortical thickness was observed in temporal and frontal areas; smaller size of some parts of hippocampus structure was found in suicidal patients.

Volumetric brain analysis in our study revealed that frontal cortex thickness was reduced in rostral middle frontal region on the left hemisphere and superior frontal regions on both hemispheres. Moreover, these differences in cortical thickness were significantly higher in the assessment of patients after repeated suicide attempts: the significant difference in cortical thinning of almost all frontal regions was found.

Many studies investigated frontal cortex role in suicidality. It is known that DL, VL areas of PFC and ACC regulate behaviour and emotions [15]. The aforementioned changes lead to impaired behavioural control and impulsive behaviour. It is thought that in an emotionally unstable person the frontal cortex limbic system inhibition is not sufficient enough, which causes impulsive, irrational decision making and emotional liability in individuals $[16,17]$. Serotonin also plays an important role in emotion regulation and behavioural control. People with serotonin deficiency can succumb to strong emotions like suicidal thoughts and aggressive actions that ultimately lead to suicide [18]. In the findings of other researchers, cortex thinning in PFC and OFC areas was linked with suicidality $[19,20]$. Atrophy in prefrontal cortex ventrolateral, dorsolateral and anterior cingulate parts was also discovered in MDD, bipolar depression and schizophrenia patients who attempted suicide $[4,5,21]$. Study of Wang et al. found that MDD patients with a history of SA had a reduced volume in the right and left amygdala and ventral, medial, dorsal PFC. These demonstrate the role of the amygdala and PFC in the pathogenesis of suicidal behavior and imply the amygdala-PFC circuit as a probable target for detection and prevention of suicidal behavior [22]. 
Our study has also revealed significant differences in superior frontal gyrus, rostral middle frontal gyrus, pars triangularis and precentral gyrus and other areas of frontal cortex. It has been investigated that pars triangularis is associated with cognitive control of memory and may contribute to suicidal behaviour through a connection with hippocampus [23]. Prefrontal cortex is also linked to suicidality because of its role in executive functions [24]. Other frontal cortex regions involved in suicide have not yet been investigated.

Furthermore, our investigation of temporal lobe structures revealed reduced cortical thickness in inferior, middle and superior regions in patients with first suicidal attempt and nearly all TC regions - in patients with repeated suicidal attempts. It is known about the association of TC with limbic structures and the PFC: these interfaces are important in recognizing and controlling mood and emotion [25]. Superior temporal gyrus is related to emotional intelligence, the ability of following own and others' emotions to make decisions and perform actions [15]. Superior temporal gyrus and sulcus are the components of the face recognition system [26]. This gyrus becomes active upon seeing scared faces [27]. Superior temporal sulcus, amygdala and insula analyse movements of other objects, providing information about their intentions [28]. In addition, these structures regulate a sudden, reflexive response to negative visual stimuli [29]. In other studies, the role of TC in suicidality has been mainly linked to degradation in medial and superior cortices $[5,20,27,30,31]$. This could be due to the fact that temporal lobe contributes to emotion responding [32]. Our research has demonstrated significant volumetric results in the previously mentioned middle, superior TC and also in inferior, left fusiform and left temporal pole. Similar findings were reported in other studies that investigated fusiform relation to borderline personality disorder, while smaller temporal pole is associated with psychotic disorders and suicide attempts [27, 33].

One of the aims of our study was to examine hippocampus structure differences in suicide attempted patients. When comparing all study patients and healthy controls, we have found significant volume differences in some left and right parts of hippocampus structures. However, some hippocampus regions were found as reduced only in patients after repeated SA, without significant differences in patients after first suicidal attempt in comparison to controls.

The hippocampus is associated with the pathophysiology of mental disorders; the main function of the hippocampus is memory processing. Changes in this cognitive function are associated with suicidal behaviour and lower hippocampal volume in individuals who have attempted suicide [34]. It also modulates the activity of the hypothalamic-pituitary-adrenal axis which is impaired in people who have attempted suicide, not to mention the prefrontal cortex which perform executing functions and is regulated by the hippocampus $[35,36]$. An acute stressing event and high levels of cortisol also could associate with decreased volume of the hippocampus [37]. However, hippocampus volume changes in suicidal behavior remained contradictory [38].

Our findings support the Colle et al. study in which depressed suicide patients had smaller right and total hippocampus volume than non-suicidal attempters. In addition, researchers have suggested that hippocampal volume could be a suicidal state marker in depressive disorder [39]. Hippocampal volumes 
were negatively associated with impulsivity in individuals; high lethality attempts were associated with smaller volumes of the hippocampus and parahippocampal gyrus in adult suicidal attempters $[27,40]$. Other study of Gosnell and colleagues has found smaller hippocampus in depressed suicidal patients in comparison to healthy controls. However, they did not find differences in hippocampus of suicidal and not suicidal depressive patients. Researchers hypothesized that hippocampus difference could be due to a characteristic other than suicidality [19].

It is known that mental disorder, especially MDD and schizophrenia, are related to suicide behaviors [41, 42]; affective disorders comprise more than half of all suicide deaths [43]. Most of the volumetric studies evaluating suicidal patients provided observations when comparing suicidal vs. non-suicidal patients with an affective or psychotic disorder. In our study only one-third of patients were diagnosed with MDD, other patients had adjustment disorder, anxiety disorders and personality disorders. However, our study results revealed that reduced brain volume in suicidal patients is completely unrelated to neither age, gender, method of suicide attempt or diagnosis of MDD. Hence, at this stage of understanding, we hypothesized that the model of suicidal behavior is related to the reduction of brain volume in specific brain regions - frontal, temporal cortex and hippocampal structures. Moreover, these changes become express with repeated suicidal attempts.

Our study has a few limitations. For brain structure visualization we used 1,5T tomography imaging. 3T computer tomography has a better resolution because of the ability to better visualize the boundaries between grey matter, white matter, and cerebrospinal fluid. However, the 1.5T tomography imaging provides a more accurate measurement of realistically small brain structures such as the hippocampus, amygdala, and nuclei [44]. An apparent limitation of the method is a cross-sectional design. This bears importance because differentiating the cause and effect becomes impossible - we are unable to tell whether reduced brain volume contributes to suicidal behaviour or the opposite. The prospective analysis of structural brain changes after each repeated SA could provide more clear information about neuroimaging markers of the suicidal brain.

\section{Conclusions}

Suicide attempters had lower cortical thickness in frontal and temporal brain regions and smaller parts of hippocampus than healthy subjects without history of suicide attempt and mental disorder. These differences were found independently to patients' age, gender or diagnosis of depressive disorder. The atrophy of frontal, temporal cortex and hippocampus parts were significantly higher in repeated suicide attempters than in first suicide attempters. These findings suggest that repeated suicidal behavior is associated with intensifying changes in specific brain structures.

\section{Abbreviations}

ACC - anterior cingulate cortex 
CA - cornu amonis

DL - dorsolateral

EU - European Union

First SA group - suicide attempters with a history of at least one suicide attempt

GC-DG - granule cell layer of dentate gyrus

HATA - hippocampus-amygdala transition area

$\mathrm{HC}$ - healthy controls

MDD - major depressive disorder

ML - molecular layer

MRI - magnetic resonance imaging

OFC - orbitofrontal cortex

PFC - prefrontal cortex

SA - suicide attempt

SA $>1$ - more than one suicide attempt

SA>1 group - suicide attempters with more than one suicide attempt during the lifetime

$\mathrm{T}-$ tesla

T2W - T2-weighted

TC - temporal cortex

TW1 - T1-weighted

VL - ventrolateral

\section{Declarations}

\section{Ethics approval and consent to participate}

This study was approved by the Kaunas Regional Biomedical Research Ethics Committee (approval no. BEC-LSMUCR-67; 2017-06-23). 


\section{Consent for publication}

All study patients and controls signed informed consent to participate in the study.

\section{Availability of data and materials}

The datasets used and analyzed during the current study are available to be collected from the corresponding author on reasonable request.

\section{Competing interests}

The authors declare that they have no competing interests.

\section{Funding}

No funding was received for this study.

\section{Authors' contributions}

VS, RG and VA designed the study and wrote the protocol. VS and KD coordinated the study, performed the selection of the subjects and psychiatric evaluation, collected and analyzed the data. RG organized MRI brain scans and analyzed the data. MS performed volumetric brain analysis using FreeSurfer. VS, MS and NR analyzed the data and performed the statistical analysis. MS and VS wrote the first draft of the manuscript. All authors critically revised the draft and gave the approval for final manuscript.

\section{Acknowledgements}

The authors wish to thank all patients and control subjects for participating in the study, as well as, radiology technicians from Radiology department for performing MRI scans.

\section{Authors' Information}

MS, MD, resident at the Department of Radiology of Lithuanian University of Health Sciences.

RG, PhD, doctor-radiologist and Professor at the Department of Radiology of Lithuanian University of Health Sciences.

VA, PhD, doctor-psychiatrist, Professor and the head of Psychiatry Clinic at Lithuanian University of Health Sciences.

$\mathrm{KD}, \mathrm{PhD}$, doctor-psychiatrist and lecturer the Department of Psychiatry at Lithuanian University of Health Sciences.

NJ, Laboratory of Behavioural Medicine, Neuroscience Institute of Lithuanian University of Health Sciences. 
VS, PhD, doctor-psychiatrist, assoc. professor at Psychiatry Clinic and senior researcher at Laboratory of Behavioural Medicine, Neuroscience Institute of Lithuanian University of Health Sciences.

\section{References}

1. WHO: Global Health Observatory (GHO) data. In: Suicide rates (per 100000 population). www.who.int/gho/mental_health/suicide_rates/en (2016). Accessed 20 Apr 2020.

2. Ludwig B, Roy B, Wang Q, Birur B, Dwivedi Y. The Life Span Model of Suicide and Its Neurobiological Foundation. Frontiers in neuroscience. 2017; 11:74.

3. Dominguez-Baleon C, Gutierrez-Mondragon LF, Campos-Gonzalez Al, Renteria ME. Neuroimaging Studies of Suicidal Behavior and Non-suicidal Self-Injury in Psychiatric Patients: A Systematic Review. Frontiers in psychiatry. 2018; 9:500.

4. Wagner G, Schultz CC, Koch K, Schachtzabel C, Sauer H, Schlosser RG. Prefrontal cortical thickness in depressed patients with high-risk for suicidal behavior. Journal of psychiatric research. 2012; 46(11):1449-1455.

5. Aguilar EJ, Garcia-Marti G, Marti-Bonmati L, Lull JJ, Moratal D, Escarti MJ, Robles M, Gonzalez JC, Guillamon MI, Sanjuan J. Left orbitofrontal and superior temporal gyrus structural changes associated to suicidal behavior in patients with schizophrenia. Progress in neuropsychopharmacology \& biological psychiatry. 2008; 32(7):1673-1676.

6. Rusch N, Spoletini I, Wilke M, Martinotti G, Bria P, Trequattrini A, Bonaviri G, Caltagirone C, Spalletta G. Inferior frontal white matter volume and suicidality in schizophrenia. Psychiatry research. 2008; 164(3):206-214.

7. Spoletini I, Piras F, Fagioli S, Rubino IA, Martinotti G, Siracusano A, Caltagirone C, Spalletta G. Suicidal attempts and increased right amygdala volume in schizophrenia. Schizophrenia research. 2011; 125(1):30-40.

8. Jovev M, Garner B, Phillips L, Velakoulis D, Wood SJ, Jackson HJ, Pantelis C, McGorry PD, Chanen AM. An MRI study of pituitary volume and parasuicidal behavior in teenagers with first-presentation borderline personality disorder. Psychiatry research. 2008; 162(3):273-277.

9. Monkul ES, Hatch JP, Nicoletti MA, Spence S, Brambilla P, Lacerda AL, Sassi RB, Mallinger AG, Keshavan MS, Soares JC. Fronto-limbic brain structures in suicidal and non-suicidal female patients with major depressive disorder. Molecular psychiatry. 2007; 12(4):360-366.

10. Association AP: Diagnostic and Statistical Manual of Mental Disorders (DSM-5 (R)). VA, United States: American Psychiatric Association Publishing; 2013.

11. Crosby A.E. OL, Melanson C. Self-directed violence surveillance: uniform definitions and recommended data elements. Atlanta, GA: Centers for Disease Control and Prevention, National Center for Injury Prevention and Control; 2011.

12. Dale AM, Fischl B, Sereno MI. Cortical surface-based analysis. I. Segmentation and surface reconstruction. Neurolmage. 1999; 9(2):179-194. 
13. Desikan RS, Segonne F, Fischl B, Quinn BT, Dickerson BC, Blacker D, Buckner RL, Dale AM, Maguire RP, Hyman BT et al. An automated labeling system for subdividing the human cerebral cortex on MRI scans into gyral based regions of interest. Neurolmage. 2006; 31(3):968-980.

14. Iglesias JE, Van Leemput K, Augustinack J, Insausti R, Fischl B, Reuter M. Bayesian longitudinal segmentation of hippocampal substructures in brain MRI using subject-specific atlases. Neurolmage. 2016; 141:542-555.

15. McCarthy CS, Ramprashad A, Thompson C, Botti JA, Coman IL, Kates WR. A comparison of FreeSurfer-generated data with and without manual intervention. Frontiers in neuroscience. 2015; 9:379.

16. Sanfilipo M, Lafargue T, Rusinek H, Arena L, Loneragan C, Lautin A, Feiner D, Rotrosen J, Wolkin A. Volumetric measure of the frontal and temporal lobe regions in schizophrenia: relationship to negative symptoms. Archives of general psychiatry. 2000; 57(5):471-480.

17. Shamay-Tsoory SG, Harari H, Aharon-Peretz J, Levkovitz Y. The role of the orbitofrontal cortex in affective theory of mind deficits in criminal offenders with psychopathic tendencies. Cortex; a journal devoted to the study of the nervous system and behavior. 2010; 46(5):668-677.

18. Joiner TE, Jr., Brown JS, Wingate LR. The psychology and neurobiology of suicidal behavior. Annual review of psychology. 2005; 56:287-314.

19. Gosnell SN, Velasquez KM, Molfese DL, Molfese PJ, Madan A, Fowler JC, Christopher Frueh B, Baldwin PR, Salas RPrefrontal cortex, temporal cortex, and hippocampus volume are affected in suicidal psychiatric patients. Psychiatry research:Neuroimaging. 2016; 256:50-56.

20. Giakoumatos Cl, Tandon N, Shah J, Mathew IT, Brady RO, Clementz BA, Pearlson GD, Thaker GK, Tamminga CA, Sweeney JA et al. Are structural brain abnormalities associated with suicidal behavior in patients with psychotic disorders? Journal of psychiatric research 2013, 47(10):1389-1395.

21. Ding Y, Lawrence N, Olie E, Cyprien F, le Bars E, Bonafe A, Phillips ML, Courtet P, Jollant F. Prefrontal cortex markers of suicidal vulnerability in mood disorders: a model-based structural neuroimaging study with a translational perspective. Translational psychiatry. 2015; 5:e516.

22. Wang L, Zhao Y, Edmiston EK, Womer FY, Zhang R, Zhao P, Jiang X, Wu F, Kong L, Zhou Y et al. Structural and Functional Abnormities of Amygdala and Prefrontal Cortex in Major Depressive Disorder With Suicide Attempts. Frontiers in psychiatry. 2019; 10:923.

23. Badre $D$, Wagner AD. Left ventrolateral prefrontal cortex and the cognitive control of memory. Neuropsychologia. 2007; 45(13):2883-2901.

24. Fuster JM, Bressler SL. Past makes future: role of pFC in prediction. Journal of cognitive neuroscience. 2015; 27(4):639-654.

25. Lindquist KA, Wager TD, Kober H, Bliss-Moreau E, Barrett LF. The brain basis of emotion: a metaanalytic review. The Behavioral and brain sciences. 2012; 35(3):121-143.

26. Takeuchi H, Taki Y, Sassa Y, Hashizume H, Sekiguchi A, Fukushima A, Kawashima R. Regional gray matter density associated with emotional intelligence: evidence from voxel-based morphometry. Human brain mapping. 2011; 32(9):1497-1510. 
27. Soloff PH, Pruitt P, Sharma M, Radwan J, White R, Diwadkar VA. Structural brain abnormalities and suicidal behavior in borderline personality disorder. Journal of psychiatric research. 2012; 46(4):516525.

28. Radua J, Phillips ML, Russell T, Lawrence N, Marshall N, Kalidindi S, El-Hage W, McDonald C, Giampietro V, Brammer MJ et al. Neural response to specific components of fearful faces in healthy and schizophrenic adults. Neurolmage. 2010; 49(1):939-946.

29. Allison T, Puce A, McCarthy G. Social perception from visual cues: role of the STS region. Trends in cognitive sciences. 2000; 4(7):267-278.

30. Benedetti F, Radaelli D, Poletti S, Locatelli C, Falini A, Colombo C, Smeraldi E. Opposite effects of suicidality and lithium on gray matter volumes in bipolar depression. Journal of affective disorders, 2011; 135(1-3):139-147.

31. Pan LA, Ramos L, Segreti A, Brent DA, Phillips ML. Right superior temporal gyrus volume in adolescents with a history of suicide attempt. The British journal of psychiatry : the journal of mental science. 2015; 206(4):339-340.

32. Buhle JT, Silvers JA, Wager TD, Lopez R, Onyemekwu C, Kober H, Weber J, Ochsner KN. Cognitive reappraisal of emotion: a meta-analysis of human neuroimaging studies. Cereb Cortex. 2014; 24(11):2981-2990.

33. Harenski CL, Brook M, Kosson DS, Bustillo JR, Harenski KA, Caldwell MF, Van Rybroek GJ, Koenigs M, Decety J, Thornton DM et al. Socio-neuro risk factors for suicidal behavior in criminal offenders with psychotic disorders. Social cognitive and affective neuroscience. 2017; 12(1):70-80.

34. Richard-Devantoy S, Berlim MT, Jollant F. A meta-analysis of neuropsychological markers of vulnerability to suicidal behavior in mood disorders. Psychological medicine. 2014; 44(8):1663-1673.

35. Jollant F, Lawrence NL, Olie E, Guillaume $S$, Courtet $P$. The suicidal mind and brain: a review of neuropsychological and neuroimaging studies. The world journal of biological psychiatry : the official journal of the World Federation of Societies of Biological Psychiatry. 2011; 12(5):319-339.

36. Mann JJ, Currier D, Stanley B, Oquendo MA, Amsel LV, Ellis SP. Can biological tests assist prediction of suicide in mood disorders? The international journal of neuropsychopharmacology. 2006; 9(4):465-474.

37. Moica T, Grecu IG, Moica S, Grecu MG, Buicu GE: Cortisol and Hippocampal Volume as Predictors of Active Suicidal Behavior in Major Depressive Disorder: Case Report. Balkan medical journal. 2016; 33(6):706-708.

38. Chen F, Bertelsen AB, Holm IE, Nyengaard JR, Rosenberg R, Dorph-Petersen KA. Hippocampal volume and cell number in depression, schizophrenia, and suicide subjects. Brain research. 2020; 1727:146546.

39. Colle R, Chupin M, Cury C, Vandendrie C, Gressier F, Hardy P, Falissard B, Colliot O, Ducreux D, Corruble E. Depressed suicide attempters have smaller hippocampus than depressed patients without suicide attempts. Journal of psychiatric research. 2015; 61:13-18. 
40. Soloff P, White R, Diwadkar VA. Impulsivity, aggression and brain structure in high and low lethality suicide attempters with borderline personality disorder. Psychiatry research. 2014; 222(3):131-139.

41. Bachmann S: Epidemiology of Suicide and the Psychiatric Perspective. International journal of environmental research and public health. 2018; 15(7).

42. Qin P: The impact of psychiatric illness on suicide: differences by diagnosis of disorders and by sex and age of subjects. Journal of psychiatric research. 2011; 45(11):1445-1452.

43. Chesney E, Goodwin GM, Fazel S. Risks of all-cause and suicide mortality in mental disorders: a meta-review. World Psychiatry. 2014; 13(2):153-160.

44. Zhang X, Liu J, He B. Magnetic-resonance-based electrical properties tomography: a review. IEEE reviews in biomedical engineering. 2014; 7:87-96.

\section{Supplementary Information}

\section{Additional file 1}

DOC file extension

Title - Survey Questionnaire

The questionnaire was used to identify the sociodemographic characteristics and history of suicide attempt of all study patients.

\section{Supplementary Files}

This is a list of supplementary files associated with this preprint. Click to download.

- Additionalfile1SurveyQuestionnaire.docx 